REVISTA DE DERECHO UNED, NÚM. 9, 2011

\title{
LA APLICACIÓN DEL MARKETING EN LOS DESPACHOS DE ABOGADOS
}

\author{
MARKETING FOR LAW FIRMS
}

\author{
JACOBO NÚÑEZ MaRTíNEZ \\ Profesor Visitante Universidad Rey Juan Carlos I
}

Resumen: A diferencia de otros sectores del mundo privado, los despachos de abogados no han valorado la aplicación de técnicas de marketing como un factor de desarrollo en su actividad debido a las peculiares características que tiene el ejercicio de la abogacía.

No obstante la actual crisis financiera, que ha afectado de manera significativa la facturación de muchos bufetes y los cambios que se han producido en el mundo del derecho en los últimos años, está obligando a un replanteamiento de esta visión.

En este artículo se analizan las causas que están motivando la aplicación del marketing en los despachos de abogados, así como el desarrollo de un plan de marketing que se adecúe a las características propias del mundo del derecho.

Palabras clave: Marketing, Derecho, despachos de abogados, clientes, crisis, fidelizar

Abstract: Unlike other parts of the private sector, law firms have not valued marketing techniques as a catalyst for growth because of the peculiar characteristics of law practice. However, the present financial crisis that has significantly impacted the revenues of many firms, as well as the changes that have taken place in the world of law in recent years, is causing firms to consider marketing. This article analyses the factors motivating of the application of marketing techniques by law firms, as well as the development of a marketing plan adapted to the characteristics of legal sector. 
Key words: Marketing, law, law firms, customers, crisis, loyalty.

Sumario: I. Introducción.-II. Causas por las cuales se debe aplicar el Marketing jurídico.-II.1. Causas Estructurales.-II.2. Situación económica actual.-III. Aplicación del marketing jurídico.-IV. Modelos actualmente utilizados para la captación de clientes.-IV.1. El boca oído.-IV.2. Excelencia Académica.-IV.3. Otras formas de comunicación.-IV.4 Inserción anuncios en prensa.-IV.5 Nuevas tecnologías.-V. Aplicación de técnicas de marketing en los despachos de abogados.-VI. Planificación del plan de marketing.-VII. Estrategias de crecimiento desde el punto de vista del marketing.-VIII. El cliente.-VIII.1. Entrevista.-VIII.2. Fidelización del cliente.-IX. El marketing interno.-IX.1. La fidelización desde el punto de vista del marketing.-Conclusiones.

\section{INTRODUCCIÓN}

La aplicación de las técnicas de marketing en el ámbito privado se considera desde hace décadas tan esencial para el desarrollo de su actividad como lo pueden ser la gestión financiera o de Recursos Humanos.

Pero a diferencia de otros sectores del mundo empresarial, las herramientas del marketing no ha tenido la misma valoración en el sector jurídico debido a las connotaciones negativas que el vocablo marketing posee, al ser asimilado como publicidad o venta.

Las razones a este rechazo debemos buscarlas en aquellos factores intrínsecos del ejercicio de la abogacía, que siempre se ha considerado más cercana al mundo académico que al de la empresa y por tanto ha incentivado la investigación académica como factor de excelencia y soporte de la carrera jurídica en detrimento del desarrollo de otras actividades más propias del sector privado.

Tal y como indica Martínez Selva (1999) para muchos letrados el único marketing posible es aquel que se obtiene como consecuencia de su historial profesional y por tanto, es resultado de la publicidad realizada por aquellos clientes que han utilizado sus servicios.

A esta percepción debemos añadirle, al igual que ocurrió en otros países del entorno, las fuertes restricciones legales aplicadas a la publicidad como consecuencia del código deontológico en el ejercicio de la abogacía ${ }^{1}$ que prohibía a los abogados la realización de cualquier

1 Tal y como queda reflejado en el artículo 13 del Estatuto general de los Colegios 
actividad publicitaria ${ }^{2}$, y que fue parcialmente derogada como resultado del artículo 25 del nuevo estatuto de la Abogacía Española ${ }^{3}$ al incorporar una regulación más permisiva e introducir particularidades haciendo una remisión genérica a la Ley General de Publicidad y a la Ley de Competencia Desleal.

Pero en contra de la opinión generalizada que equipara al marketing con la publicidad; este axioma no es cierto, ya que si bien la publicidad es su actividad más conocida, el marketing englobaría un mayor arco de actuación puesto que su objetivo principal no es otro que el de anticiparse a las demandas de los consumidores y ofertarles aquellos servicios o productos que el cliente requiere ${ }^{4}$.

Por ello Kotler (1992) indica que el marketing se fundamenta sobre cuatro pilares básicos: la definición del mercado, la orientación al cliente, la coordinación y la rentabilidad.

Este enfoque tiene como punto de partida el intercambio centrado en el mercado, orientado hacia el consumidor y dirigido a través de la coordinación a efectos de generar satisfacción como llave para cumplir los objetivos de la organización. Por este motivo para que la organización consiga sus objetivos, necesita previamente identificar las necesidades y los deseos del público objetivo y entregar los satisfactores deseados de una forma más efectiva y eficiente que la competencia.

La American Marketing Association (AMA) define el marketing como: la función de la organización y un conjunto de procesos para crear, comunicar y entregar valor a los clientes y para mejorar las relaciones con estos últimos de manera que se beneficie toda la organización.

En el caso concreto del derecho, la función del marketing sería orientar al bufete hacia su mercado potencial a través de una mejora en los servicios que oferta, de tal forma que sea capaz de aportar un

de Abogados de España (1943) o el art. 31, a del Estatuto General de la Abogacía de 24 de junio de 1992 o con algunas variaciones en el art. 9.1 del Código de Deontología de la Abogacía Española, de 30 de junio de 1995.

2 Con la excepción de la colocación de una placa que indicase la existencia de un despacho de abogados en el inmueble.

3 Aprobado por Real Decreto 658/2001 de 22 de junio.

${ }_{4}$ Tal y como lo define uno de sus padres (Jerome Macarthy): el Marketing es la realización de aquellas actividades que tienen por objeto cumplir las metas de una organización, al anticiparse a los requerimientos del consumidor o cliente y encauzar un flujo de mercancías aptas a las necesidades y servicios que el productor presta al consumidor o cliente. 
mayor valor al cliente que sus competidores sin que para ello tenga que recurrir al precio o a la erosión de sus márgenes. Para Francesc Domínguez (2003) la finalidad básica del marketing jurídico es crear y conservar clientes, diferenciar al despacho de la competencia y construir una marca del abogado o del bufete.

Por ello es errónea la creencia de que el marketing jurídico se circunscribe únicamente a realizar una comunicación efectiva del despacho ya sea a través de la inserción de anuncios en prensa o por medio de páginas web, pues como indica Eugenia Navarro $(2011)^{5}$ la comunicación es sólo una herramienta que el marketing utiliza para transmitir un cúmulo de estrategias y por ello en muchas ocasiones no tiene el resultado deseado dado que previamente no se han planificado unos objetivos concretos y un plan de acción determinado que orienten al despacho a sus potenciales clientes ${ }^{6}$.

\section{CAUSAS POR LAS CUALES SE DEBE APLICAR EL MARKETING JURÍDICO}

Desde hace algunas décadas el sector de la abogacía se ha visto avocada a una serie de transformaciones que la han obligado a un replanteamiento de su tradicional modelo de negocio ya que enfoques y percepciones válidos hasta hace relativamente poco tiempo, han sido modificados como resultado de un aumento en la competitividad de los despachos, una reducción del mercado y un cambio en la mentalidad de los clientes cada vez es más exigentes y conscientes de su poder sobre el despacho al disponer de una oferta más amplia de elección y que por tanto buscan una mayor especialización y un trato más personalizado.

Por ello consideramos que una nueva forma de gestión centrada en el marketing se adapta mejor a la realidad actual, donde la competitividad obliga al desarrollo de nuevos modelos gerenciales que permitan la subsistencia en un mundo empresarial regido exclusivamente por las leyes del mercado y en el que es necesario un enfoque más estratégico de la práctica del derecho, aspecto donde tradicionalmente los abogados han mostrado un déficit pese a estar obligados

\footnotetext{
5 Entrevista en el Diario Expansión (fecha 04-03-11).

${ }^{6}$ En referencia al concepto de venta y publicidad en el campo del derecho Francesc Domínguez (2004) indica que respecto a la publicidad es muy discutible su utilización por los bufetes, dado que no refuerza la credibilidad, el prestigio y la reputación de los abogados del despacho.
} 
a generar negocio; y los conocimientos en derecho no proporciona de manera automática capacidad para dirigir una empresa de servicios jurídicos.

A continuación vamos a indicar, de manera esquemática, cuáles consideramos que son las principales causas que motivan la implantación de herramientas de marketing en los despachos de abogados:

\section{II.1. Causas Estructurales}

Según el último estudio publicado por el Consejo General de la Abogacía Española (2008) ${ }^{7}$ España es uno de los países de Europa con una mayor densidad de abogados por habitante (2,63 por cada 1000) muy por encima de la media europea y únicamente superado por países como Grecia ${ }^{8}$, Italia ${ }^{9}$ o Luxemburgo ${ }^{10}$.

Esta situación lejos de equiparase con la media europea, aumenta anualmente de manera progresiva de tal forma que en los últimos 10 años se ha producido un incremento del $24,5 \%$ respecto de las década anterior lo que supone una tasa de crecimiento anual del 2,5\% muy por encima del crecimiento medio total de la población activa en España que es del 3\%.

Dicho escenario es todavía más significativo, si analizamos su distribución geográfica, ya que un 68\% de los mismos ejercen la abogacía únicamente en cuatro comunidades autónomas ${ }^{11}$, que marcan una densidad de 5,14 abogados por cada 1000 habitantes $^{12}$.

Otro aspecto a destacar, según la última encuesta publicada, es que nos encontramos ante una población de abogados significativamente joven, puesto que el $75 \%$ de los colegiados ejercientes es menor de 45 años, situándose la media de edad en los 39 años.

Este dato nos indica que no solamente no se va a producir un cambio de tendencia, sino que posiblemente la densidad aumente en un futuro.

${ }^{7}$ La Abogacía Española en datos y cifras 2008. Consejo general de la Abogacía Española.

8 3,24 por cada 1000 habitantes.

93,06 por cada 1000 habitantes.

102,75 por cada 1000 habitantes.

11 Madrid, Cataluña, Valencia y Andalucía.

12 Sólo en Madrid (dato 2010) existe un total de 54.216 abogados con un aumento de 3.457 abogados con respecto al 2009. 
La globalización ha afectado de manera significativa a los despachos españoles con la llegada de bufetes internacionales, fundamentalmente de origen angloamericano, que se han consolidado en muy pocos años de una manera notable, en especial en los primeros puestos del Ranking de facturación. Estos nuevos despachos han traído consigo nuevas formas de gestión que incluyen la prestación de diversos servicios (no exclusivamente jurídicos) y que dan un servicio más global al cliente y por tanto más satisfactorio.

La internalización ha supuesto también la llegada de nuevos abogados de origen extranjero ejercientes en España y que en la actualidad suponen el 0,7\% del total de los abogados en España.

\section{II.2. Situación económica actual}

La actual crisis económica ha afectado de manera significativa a los despachos de abogados y que ha obligado a adecuarse, en muchos casos de manera dramática, a las actuales necesidades del mercado ${ }^{13}$.

Las primeras consecuencias de la crisis ha sido la caída de la facturación del sector ${ }^{14}$ y por tanto el aumento en el número de despidos en los distintos despachos que comenzó en el año 2008 y que se ha seguido produciendo a lo largo de estos años, aunque de manera más selectiva, y que según indica el periódico Expansión (2011) se seguirán produciendo a lo largo del 2012 debido a la necesidad de los bufetes de adecuar sus plantillas en relación a la prestación de sus servicios con la oferta.

Esta situación de despidos no ha sido óbice para que los grandes despachos sigan contratando nuevos miembros en sus plantillas, aunque se ralentizó la rotación y el mercado de fichajes se paró casi en su totalidad, con excepción en la contratación de juniors debido, tal y como el periódico Expansión (2011), a que nos encontramos ante la última generación de licenciados que podrán colegiarse sin necesidad de cursar el nuevo programa de máster que la ley hará obligatorio a partir del mes de noviembre y que ha provocado incerti-

${ }^{13}$ Una encuesta realizada por la Método 3 (2009) indicaba que el 29\% de los encuestados creían que la crisis había afectado seriamente a la facturación de los despachos, el 59\% afirmaba que los precios se habían estacionado y un 30\% que habían bajado.

${ }_{14}$ Si bien los grandes despachos han obtenido beneficios en este último año (2011) en especial los cinco primeros despachos de este país; no ha ocurrido lo mismo en relación a los despachos medianos o pequeños donde los efectos de la crisis se están haciendo notar de una forma más acentuada. 
dumbre ante el futuro marco de acceso a la profesión por lo que los despachos han decidido adecuarse a esta situación a fin de asegurarse una cantera suficiente ante la inseguridad de los próximos años, donde se estima que pueden existir dificultades debido a que es posible que exista una menor promoción de los mismos.

Otro factor que ha fomentado la contratación es consecuencia de la incorporación de asociados y socios en los grandes bufetes pero que a diferencia de años anteriores, donde la mayoría de los mismos procedía de la propia estructura del despacho, en la actualidad provienen tanto de la judicatura como de abogados de otras firmas que aportan una cartera de clientes considerables.

Otra consecuencia relacionada con la crisis se referiría al sueldo medio del abogado que o bien en muchos casos ha sido congelado o ha disminuido con respecto a años anteriores; siendo esta situación más patente en el caso de los junior en su primer año de contratación.

\section{APLICACIÓN DEL MARKETING JURÍDICO}

Ante la situación expuesta, la estrategia de muchos bufetes se ha centrando tanto en realizar un análisis del mercado ${ }^{15}$ a fin de encontrar nuevas oportunidades de negocio, como en optimizar aquellos servicios que más pueden demandar en la actualidad los posibles clientes. Es decir se está produciendo una orientación hacia el mercado.

De este modo, grandes y medianos despachos y en función de las actuales circunstancias, están impulsando áreas como el laboral o el procesal-penal a fin de obtener una mayor rentabilidad en detrimento del mercantil o el matrimonial hasta hace algún tiempo consideradas como motores económicos de los mismos.

Al tener cada despacho características propias, no es objeto de este artículo definir cómo se debe realizar un plan de marketing concreto, pues las necesidades e intereses de un gran despacho difieren de las de un despacho mediano y son sustancialmente distintas de uno pequeño, donde la realización de un plan de marketing se sinte-

${ }^{15}$ Cada vez más los grandes despachos están invirtiendo en departamentos específicos de comunicación y marketing dentro de sus propias estructuras a fin de fomentar el área de negocio del derecho, destacando entre ellos: Cuatrecasas Gonçalves Pereira, Garrigues, Clifforf Chance, Uría Méndez, Roca Juyent o Gómez-Acebo \& Pombo. 
tizaría en una serie de actividades concretas y que se pueden especificar de una forma más sencilla.

Un especialista en marketing únicamente ofrece pautas y diseña estrategias, pero no es quien se expone profesionalmente al mercado. Quien lo hace son los abogados y es por tanto absolutamente necesario la involucración directa del titular del bufete; ya que toda la orientación que se planee descansará en el conocimiento, experiencia profesional y en las características específicas que tenga el despacho que dirige.

Debemos indicar que el marketing jurídico si bien no es la panacea que solucionará los problemas del bufete, al menos canalizará, a través de un conjunto de acciones y herramientas, una mejora en su gestión que permitirá al despacho descubrir qué valor puede ofertar al mercado y que ha podido permanecer oculto, y en función de esas ventajas tener claro que necesidades quiere cubrir y por tanto delimitar su mercado en función de las ventajas que puede ofertar, marcando así diferencias con el resto de su competidores.

\section{MODELOS ACTUALMENTE UTILIZADOS PARA LA CAPTACIÓN DE CLIENTES}

Dado que sin la existencia de clientes no existe actividad económica, una de las premisas principales de los bufetes será la captación de nuevos clientes y la consolidación de los clientes ya existentes.

La práctica habitual hasta hace pocos años para la atracción de futuros clientes, residía esencialmente en dos formas de actuación: el boca-oído y el prestigio académico.

\section{IV.1. El boca-oído}

La trayectoria profesional es la mejor garantía para la contratación de los servicios jurídicos; esta afirmación describe en una situación real ${ }^{16}$ e inmejorable cuando se trata de un abogado de reconocido prestigio y con suficientes años de experiencia; pero dicha premisa no es aplicable a un gran número de abogados en ejercicio que por distintas circunstancias o bien no han podido demostrar su valía

16 Según el último estudio de la Abogacía, un 77\% de las personas que eligen por primera vez a un abogado, lo hacen en función consultas previas a familiares o amigos que les indican a cual deben acudir. 
profesional como consecuencia de no haber obtenido casos de suficiente relevancia que les permitiese un prestigio acorde con sus conocimientos o, simplemente, debido a que sus años de ejercicio no ha sido suficientes para consolidación de una cartera de clientes.

Si bien reiteramos que se trata de la mejor vía de comercialización posible, no por ello se encuentra exenta de peligros puesto que puede ocurrir que bien los casos que entren sean de escasa cuantía o simplemente se limiten a consultas técnicas; no podemos olvidar que un sector tan competitivo como el jurídico, cada vez es más frecuente la aparición de nuevos despachos que se adelantan a las demandas de nuestros clientes ofertándoles un servicio más acorde con sus necesidades y a un precio inferior al nuestro en una sociedad donde la fidelidad del cliente es cada día más escasa y el factor precio actúa como elemento determinante. Por este motivo, depender únicamente de esta vía de contratación puede ser peligroso, salvo que se trate de un despacho verdaderamente consolidado.

\section{IV.2. Excelencia académica}

El prestigio académico ha sido práctica común del gran abogado español dado que a través de la publicación de artículos académicos y la impartición de conferencias o clases magistrales, se consideraba que aumentaba el prestigio del letrado como consecuencia del nexo de unión existente entre la universidad y la abogacía.

Es erróneo considerar que la inclusión de artículos en medios académicos es la mejor forma de publicitarse ante nuestros clientes, ya que si bien es importante comunicar a nuestros colegas los conocimientos que poseemos sobre una materia determinada, debemos tener en cuenta que la gran mayoría de nuestros potenciales clientes o no tienen acceso a revistas especializadas, o no están interesados en las mismas, o simplemente no disponen de suficientes conocimientos técnicos para evaluar la calidad de un artículo.

En este sentido Cavero (2011) indica en una de sus once reglas del marketing jurídico que «es más sencillo demostrar que se tiene un buen servicio, que afirmar que se tiene excelencia académica». Un cliente puede percibir mucho más el servicio o la atención que le prestan que de los conocimientos jurídicos que se posean. 


\section{IV.3. Otras formas de comunicación}

Como consecuencia de la modificación legal referente a la publicidad en los despachos de abogados, las nuevas vías para la captación de clientes, en términos generales, han sido las siguientes: inserción de anuncios en la prensa y aparición en listados de abogados o la realización de páginas web.

\section{IV.4. Inserción anuncios en la prensa}

La inserción de anuncios en la prensa o la aparición del nombre del bufete en los listados tienen como finalidad mostrar al futuro usuario la especialidad y la dirección del despacho. A efectos de marketing, el problema de este tipo de publicidad reside en que es difícil que un cliente pueda apreciar aquellos matices que le motiven a la contratación de dichos servicios ${ }^{17}$, pues un anuncio publicitario no transmite la valía profesional de un despacho.

Según la última encuesta publicada por la Agencia Española de la Abogacía (2008) la forma de captación de clientes a través de la inserción de anuncios es prácticamente nula, ya que la contratación de los servicios a través de su búsqueda en listados como pueden ser las páginas amarillas supone un $0 \%$, así como la publicidad a través de la inserción de anuncios en la prensa a supuesto apenas un 3\% del total de contratación.

\section{IV.5. Nuevas tecnologías}

En la actualidad la gran mayoría de los despachos disponen de páginas web donde ofertan sus servicios profesionales.

La aparición de páginas web han permitido a los bufetes no solamente la posibilidad de publicitarse, sino también la capacidad de que potenciales clientes puedan conocer aspectos más concretos de la gestión del despacho, como pueden ser su filosofía o la metodología de trabajo.

17 Tipo de servicio, cobertura, atención, tratamiento de los casos, etc...

18 Si buscados abogados España en los servidores más conocidos, tal y como podría utilizar cualquier potencial clientes, como pueden ser google y yahoo, encontramos que el primero existen 14.1000 .000 entradas y en el segundo 35.900 .000 entradas por despachos de abogados en España. 
El aspecto negativo de la utilización exclusiva de esta vía de comunicación para la atracción de clientes radica en el gran número de páginas web existentes en la actualidad que ofertan los mismos servicios jurídicos que nuestro despacho ${ }^{18}$ y que dificultan que un futuro cliente de manera circunstancial pueda acceder de una manera directa a nuestra página, salvo que nos posicionemos en las primeras páginas del servidor; lo cual para un despacho mediano o pequeño es muy complicado al encontrarse copadas por las principales firmas del país.

Desconocemos el grado de atracción que han tenido las páginas web como motor de contratación de servicios jurídicos, ya que la última encuesta publicada por la Agencia Española de la Abogacía no proporciona datos al respecto.

Creemos que la efectividad de una página web se produce una vez que el futuro cliente ha tenido conocimiento de la existencia del despacho de abogados. Puesto que el 77\% de los clientes acceden a través de una recomendación de algún conocido o familiar, es lógico pensar que buscarán en la red el nombre del despacho con la idea de conocer más el bufete antes de su contratación; por este motivo la importancia de la página web radica en la imagen que se proyecte al futuro cliente, pues una página web con un mal diseño o desactualizada puede desincentivar al potencial cliente.

\section{APLICACIÓN DE TÉCNICAS DE MARKETING EN LOS DESPACHOS DE ABOGADOS}

El marketing jurídico se englobaría dentro del denominado marketing de servicios debido a las especiales características del mismo ${ }^{19}$; y dada su complejidad, Grönroos (1990) sugiere que además de las 4 $\mathrm{ps}^{20}$ aplicables al marketing tradicional, se deben añadir dos herramientas más como son el marketing interactivo y el marketing interno.

Entenderíamos por marketing interactivo la habilidad para el trato con los clientes, tanto para la atracción de los mismos como en el desarrollo de la calidad y la prestación del servicio ofertado.

A modo de ejemplo, si los buscamos por especialidad, ej. Abogados civil-España: 3.900.5000, penal: 11.300.000 o simplemente por área geográfica: ej. Abogados-LeónEspaña: 3.620.000.

${ }_{19}$ Intangibilidad, inseparabilidad, implicación del usuario, variabilidad, carácter perecedero y distribución directa.

${ }^{20}$ Producto, plaza, precio y promoción. 
El Marketing interno sería aquella actividad que tiene por objeto que los abogados del bufete se identifiquen con la filosofía y los valores que el despacho oferta a los clientes y de esta forma aumentar su motivación y la fidelidad al mismo.

Dado la escasez de espacio en ese artículo nos vamos a centrar únicamente en estos dos aspectos para el desarrollo del marketing jurídico.

\section{PLANIFICACIÓN DEL PLAN DE MARKETING}

Antes de realizar cualquier plan deberemos disponer de un marco lógico que nos permita comprender los distintos condicionantes que envuelven nuestro despacho, tales como: a) el tamaño que tenemos, b) nuestra historia, c) el modelo de gestión elegido o d) cómo nos encontramos con respecto a los competidores a fin de situarnos de cara a planificar nuestra estrategia.

Este enfoque estratégico, basado en nuestra situación, va a permitirnos la creación de una renta económica en función de las capacidades específicas que poseamos y el entorno el cual nos rodea.

El procedimiento para la realización de este marco se centrará en la recolección del mayor número de datos disponible ${ }^{21}$, fundamentalmente de carácter cualitativo; en el caso de tratarse de percepciones de carácter subjetivo, éstas serán analizadas de manera sincera, crítica y evaluadas desde diferentes prismas.

Las recepción de esta información se realizará a través de dos vías: una primera vía que tendría su origen en aquella información obtenida en nuestro propio despacho a fin de obtener un análisis interno de nuestra gestión; y una segunda vía que tendría su procedencia en nuestro entorno inmediato ${ }^{22}$ dado que es el aquel que nos afecta de una manera más directa ${ }^{23}$.

21 Tal y como indica Francesc Domínguez (2003) todo bufete debe gestionar el recurso información mediante unos sistemas de información práctica que permita detecta oportunidades y actuar.

22 En el caso de un bufete mediano de una capital de provincias, los datos que nos interesarían, deberían ser el número de casos producidos en los dos últimos años, el $\mathrm{n}^{\circ}$ de despachos y su área de especialización, situación económica de la población, sector más afectado, previsiones existentes.

${ }^{23}$ Una manera efectiva para recopilar información sería a través de estadísticas ofrecidas por asociaciones profesionales, ayuntamientos, empresas privadas de servicios de información del mercado. 
Para la ordenación de los datos existen diferentes modelos testados; en este caso hemos seleccionado un modelo de auto encuesta ya que pese a su sencillez permite obtener conclusiones claras.

Debemos tener en cuenta que dependiendo del tipo de despacho y el volumen del mismo, la planificación será más compleja, así cuanto más incierto sea el futuro será más complicado.

Se ha dividido la auto encuesta en cuatro grandes bloques temáticos que serían: especialidad, modelos de gestión, clientes y competidores:

Las preguntas propuestas son las siguientes:

\section{Especialidad}

1. ¿Hacia qué áreas del Derecho está orientado nuestro bufete?

2. ¿Tenemos prestigio en nuestra especialidad?

3. ¿Aportamos algo distinto a la competencia?

4. ¿En qué situación se encuentra nuestra área de conocimiento: crecimiento, estancamiento o declive?

5. ¿Es en la actualidad un área rentable?

6. ¿Cuál es la cualificación de nuestro personal en relación con la competencia?

\section{Modelo de gestión}

1. ¿Nuestro modelo de gestión ha sido testado en otros despachos o es propio?

2. ¿Los objetivos y metas del nuestro despacho están expresados claramente?

3. ¿Se comunican los objetivos a toda la organización de forma adecuada?

4. ¿Es la estructura de la organización adecuada?

5. ¿Son efectivos los mecanismos de control y remuneración del personal?

6. ¿Emplea la empresa concepto de administración estratégi$\mathrm{ca}$ ?

\section{$\underline{\text { Clientes }}$}

1. ¿Cuál es el segmento perfil socioeconómico de los clientes? 
2. ¿Cómo hemos obtenido a nuestros clientes?

3. ¿A qué factores son sensibles nuestros clientes?

4. ¿Qué conjunto de ventajas busca el cliente?

5. ¿Podemos acceder de una forma más eficiente y económica a nuestros clientes?

6. ¿Realizamos actuaciones específicas a nuestro segmento para ser más conocidos?

7. ¿Cuál es la capacidad de retención del cliente: cuantos repiten?

8. ¿Son rentables nuestros clientes?

\section{Competidores}

1. ¿Cuál es el tipo de comportamiento competitivo dominante?

2. ¿Cuál es la cuota de mercado de los 3 o 5 competidores más directos o más importantes?

3. ¿Cuáles son los puntos vulnerables de la competencia?

4. ¿Hemos conseguido captar clientes de la competencia y viceversa?

Las respuestas a las preguntas planteadas van a permitir identificar tanto nuestras debilidades y fortalezas como encuadrar claramente el entorno en el cual actuamos.

De esta forma podremos obtener las siguientes conclusiones:

a) En primer lugar definir y descubrir aquellos servicios y ventajas propias de nuestra oferta en las cuales podemos encontrar un valor superior al de nuestros competidores, y que determinará la posición a adoptar a la hora de relacionarnos con nuestro mercado potencial.

b) En segundo lugar y en función de nuestro valor, delimitar aquel mercado en que las necesidades y demandas de este sean coincidentes con el valor que podamos aportar; para de esta forma colocarnos en una situación de ventaja con respecto a nuestros competidores.

c) En tercer lugar, y no menos importante, descubrir qué aspectos de nuestra gestión interna pueden ser mejorables y si están en sintonía con nuestro valor, ya que de no ser así, de- 
berían ser modificados a fin de potenciar nuestra ventaja competitiva.

En el caso de no haber encontrado una ventaja competitiva lo suficientemente importante para ser atrayente a los potenciales clientes, la estrategia a seguir sería la siguiente:

a) Análisis del mercado al cuál queramos centrarnos a fin de descubrir que necesidades demandan y no han sido cubiertas por la competencia.

b) Atendiendo a esas necesidades particulares de los potenciales clientes, ver si somos capaces de ofertarlas, analizando también a la competencia, en especial a aquella relacionada con nuestra situación geográfica y sus posibilidades respecto a nosotros.

c) Posteriormente focalizar todos los esfuerzos del bufete (incluida la gestión interna) para cubrir la necesidad de nuestros potenciales clientes y que no ha sido cubierta.

Debemos de tener en cuenta que para que un plan de marketing sea efectivo, tal y como indica Francesc Domínguez (2002) la base esencial pasa por tener una excelente base técnica en derecho, ya que sin calidad jurídica no hay marketing eficaz.

\section{ESTRATEGIAS DE CRECIMIENTO DESDE EL PUNTO DE VISTA DEL MARKETING}

La estrategia de crecimiento tiene como objetivo la obtención de nuevos clientes a través de: a) ofertar una mejora en servicios a clientes detectando aspectos no cubiertos por la competencia; b) buscar nuevos clientes que se adapten a las características del despacho aportando un valor que no puede dar la competencia.

Una vez identificados nuestras capacidades a través de la realización del marco lógico y conocido nuestro valor; pasaríamos a la siguiente fase del plan consistente en analizar dentro del mercado global, cuál es el segmento que mejor podemos satisfacer como consecuencia de las ventajas competitivas de nuestro despacho aportando un valor superior al de la competencia.

A la hora de seleccionar nuestro nicho de mercado debemos tener en cuenta que el mismo debe de cumplir, al menos, una serie de requisitos ya que de lo contrarío no sería efectivo. 
Las características a cumplir serían las siguientes: a) fácilmente identificable. b) medible; c) accesible; d) rentable; e) y que sus necesidades sean coincidentes con el valor ofertado por nosotros.

Tradicionalmente en el campo del derecho la selección del mercado se realizaba fundamentalmente en función de la especialidad del despacho (mercantil, laboral, penal...) si bien esa segmentación es lógica; cabe la posibilidad de realizar otro tipo de segmentación en función de diferentes variables que sean consecuencias de nuestra propia ventaja: un sector de negocios concreto, el tamaño de la empresa o un área geográfica determinada. Cabe también la posibilidad de realizar una segmentación de conjugación multivariable que pudiese ser: mercantil-mediana empresa -zona geográfica determinadainstituciones públicas.

\section{EL CLIENTE}

Una vez definido nuestro mercado objetivo comenzaremos una fase de estudio más específico referido a reconocer las demandas y necesidades concretas de nuestros potenciales clientes y encajarlas en el servicio que pretendemos ofertarles.

Por ello es necesario recopilar el mayor número de información disponible sobre aquellos clientes en los que centramos nuestros intereses, de tal forma que descubriendo sus necesidades podamos focalizar de una manera más efectiva nuestros servicios jurídicos, anticipándonos en aquellos aspectos concretos que se ajusten mejor las expectativas.

Las expectativas del cliente se fundamentarían en función de dos factores: su experiencia y las precepciones generales.

Respecto a la experiencia del cliente, y a diferencia de décadas anteriores, en la actualidad los clientes tienen un mayor nivel de información y por tanto una mayor exigencia respecto al servicio esperado.

Respecto a las percepciones, estas se incluirían de manera genérica dentro del concepto de calidad del servicio a prestar.

El término calidad es, por costumbre, un concepto abstracto, subjetivo y acompañado de un fuerte componente emocional, que será necesario demostrar de la manera más tangible posible a efectos de cubrir las percepciones del cliente. 
Para ello un bufete cuenta únicamente con dos herramientas: la marca o prestigio del mismo, que en si supone una garantía y por tanto aporta seguridad al cliente. Y un segundo elemento que se relacionaría con la calidad del servicio que se presta y por tanto con la imagen de consistencia que podamos ofertar.

Respecto a la marca ya ha sido tratada en distintos puntos de este artículo, por lo tanto no nos centraremos en el mismo.

Respecto a la calidad en el servicio, éste puede ser demostrable relacionándolo con aquellos elementos que el cliente puede apreciar como factores de excelencia.

Dicho elementos serían los siguientes:

1) Solvencia referida a los conocimientos técnicos y jurídicos sobre los servicios que ofertamos.

2) Conocimiento sobre las necesidades y demandas del cliente y capacidad de dar respuesta a las mismas.

3) Formalidad en el sentido de cumplir con las obligaciones exigidas, cumpliendo lo pactado, colocando en un lugar prioritario los intereses del cliente.

4) Evitar demoras injustificadas, así como la creación de un canal abierto con el cliente donde se le informe de los pasos y gestiones que se están realizando, el cliente tenga que preguntarnos a nosotros.

5) Buena preparación del personal de contacto, ya que la actitud de profesionalidad de los empleados repercutirá notablemente en la satisfacción del cliente y aportará capacidad de respuesta.

\section{VIII.1. Entrevista}

El letrado debe adaptarse al cliente en la reunión y por tanto su actitud y forma de expresión que deberán ser acorde con el receptor, traduciendo los posibles tecnicismos, pasos procesales, procedimientos y documentos, pero debiendo tener en cuenta en todo momento que somos nosotros los especialistas en la materia y por tanto los que podemos solucionar sus necesidades.

Por tanto la interacción estará guiada hacia la solución de los problemas que son consultados por el cliente con el fin de informar- 
le de las necesidades de su situación y aquellos servicios que pueden satisfacerle.

El objetivo por tanto es disipar las incertidumbres, una vez escuchadas las necesidades del adoptante, planteando soluciones alternativas a las mismas desde la óptica del mismo, así como anticiparse a las objeciones del cliente de forma objetiva.

\section{VIII.2. Fidelización del cliente}

La fidelización de clientes en ámbito de la abogacía siempre ha sido considerada como un aspecto complejo debido a las peculiaridades propias del servicio que se presta, pues comúnmente los ciudadanos acuden con objeto de realizar únicamente una consulta o una gestión determinada.

Así, según la última encuesta realizada por el Consejo General de la Abogacía Española (2008) el 53\% de los usuarios que acudieron a un bufete únicamente realizaron una consulta.

De aquellos usuarios que han efectuado más de una consulta, el índice de fidelización es bajo, ya que un 59\% cambió de despacho motivado por la búsqueda de una mayor especialización hacia su caso $^{24}$, y un $12 \%$ como resultado de deficiencias en el servicio ofertado. También debemos indicar, siempre según la última encuesta publicada, que el factor precio apenas supuso un 3\% como causa de abandono.

En función de los resultados expuestos consideramos que la orientación del bufete hacia la filosofía del marketing puede reducir en número de abandonos fidelizando a un mayor número de clientes que como consecuencia de las siguientes variables:

Una estrategia de marketing supone una alta implicación originaria por parte del despacho en las necesidades del cliente, lo cual, en principio, garantiza al cliente una alta especialización en los asuntos del mismo a través del profundo conocimiento de su abogado que difícilmente puede alcanzar otro despacho en poco tiempo.

La filosofía del marketing implica también trabajar con el cliente, demostrando la calidad del bufete a través de los servicios que le

${ }^{24}$ Según la última encuesta:

Buscaba a alguien especializado en el nuevo tipo de asunto (46\%); por recomendación de mi propio abogado anterior (1\%); me inspiraba más confianza para llevar el nuevo asunto (12\%). 
son ofertados. Esto obliga a una atención continua hacia las demandas del cliente que se manifiesta a través de los flujos de información que el despacho proporciona al cliente, adelantándose a sus requerimientos, e informándole de forma puntual y detallada en todo momento de la situación actual del proceso en el cual se encuentra implicado el cliente, así como de los pasos que se realizarán en los siguientes pasos del proceso.

Con una perspectiva de marketing debemos analizar las situaciones desde el punto de vista del cliente. Esto supone que como letrados y conocedores de los procedimientos judiciales en muchas ocasiones no se da importancia a determinadas partes del proceso por considerarlos automáticos, pero no ocurre lo mismo en el caso del cliente, ya que en primer lugar suele desconocer dichos procedimientos procesales y en segundo lugar su caso, por pequeño que sea, es lo más importante y por tanto exige una máxima atención.

En referencia al cambio de abogados producidos durante un proceso judicial, aquellos clientes que así lo hicieron (45 de un total de 190 que habían ido a juicio) señalaron como principales razones par efectuar el cambio «no hizo nada $(40 \%)$ y no daba confianza según un $33 \%$.

\section{EL MARKETING INTERNO}

Las empresas no se mueven en un único mercado; si no que se encuentran inmersos en tantos mercados como recursos necesiten para su funcionamiento. Uno de estos mercados es el referido a nuestros clientes internos ${ }^{25}$ puesto que de su grado de satisfacción dependerá en gran medida la satisfacción de los clientes finales.

Este hecho es todavía más significativo en el caso del derecho, ya que el principal recurso de los despachos es el talento humano y por tanto cuando un abogado abandona un bufete se pierde una parte importante del conocimiento del mismo.

Pero es todavía más dramático cuando el abogado que decide abandonarnos pertenece a la zona de socios o es responsable de un área de negocio; pues en ese caso no solo perdemos conocimiento, sino que por lo general también una parte importante de la facturación.

25 Entendido como trabajadores. 
Es por ello que en muchas ocasiones, y con objeto de retener al abogado, se suele realizar una contraoferta económica aunque en muchos casos únicamente se consiga aplazar el problema; puesto que esta actitud tiende a considerar como causa principal de la deserción razones económicas; pero esta premisa no es del todo cierta, ya que como indica Martínez Selva (1999) «la mayoría de los profesionales que cambian de trabajo lo hacen por el mal ambiente del mismo». Es por este motivo que se debe incentivar también otro tipo salarios como los denominados salarios emocionales y que Huete (2005) indica como «la capacidad de conseguir que las personas se sientan bien pagadas por su esfuerzo con algo más que dinero», así que junto a una retribución económica justa, también se trataría de desarrollar asuntos que les resulten atractivos por el tipo de materia y que se sientan preparados para prestar servicios jurídicos que resulten útiles a quien los reciba.

\section{IX.1. La fidelización desde el punto de vista del Marketing}

La estrategia de marketing intenta conseguir la total implicación de los trabajadores con los objetivos del despacho. Para ello es necesario que los trabajadores se identifiquen con los valores del despacho, que comprendan que se espera de ellos y que se les motive a fin de que puedan desarrollar las habilidades para las cuales fueron contratados.

Los elementos clave para el logro de estos objetivos son la información y el trabajo en equipo.

El factor comunicación es esencial para el buen funcionamiento de una organización ya que permite dar respuestas rápidas a los problemas que pueden surgir, explicar los procedimientos a seguir con los clientes y recibir las dudas o iniciativas que puedan tener los empleados.

Respecto al trabajo en equipo, es un aspecto más complicado debido a que nos encontramos ante una profesión donde no existe cultura de equipo, al ser un trabajo eminentemente individualista y donde únicamente existe un equipo de colaboración.

El trabajo en equipo va a permitir no solo la aportación de cada uno de los miembros en un caso concreto, sino también mantener la identidad del despacho en todos los niveles y jerarquías.

Debemos tener en cuenta que los clientes no son de nadie y el pro- 
blema de una gestión muy personalista por parte de un abogado del bufete, hace que en primer lugar que considere al cliente como suyo, y como consecuencia que el cliente identifique al abogado con el despacho y por tanto único responsable de su asunto.

Un mayor trabajo en equipo permite que el cliente pueda apreciar que la forma de producción de su servicio es consecuencia del despacho, donde se ha realizado un trabajo previo y donde la actuación y el contacto con diferentes abogados en el proceso, dificulte la marcha de determinados clientes.

\section{CONCLUSIONES}

Los cambios acaecidos en las últimas décadas en el ejercicio de la abogacía han obligado a muchos despachos a modificar su tradicional modelo de gestión a fin de poder seguir siendo competitivos y máxime ahora, donde la crisis económica está afectando de manera significativa a un alto número de bufetes.

Por este motivo la aplicación de técnicas de marketing va a posibilitar descubrir al despacho capacidades propias que han podido permanecer ocultas; así como reorientar la actividad del bufete hacia aquellos mercados que pueden demandar los servicios que oferta de una manera más efectiva.

También con la aplicación del marketing jurídico, que engloba un concepto más amplio que el de la publicidad, se pretende obtener la de fidelidad de los clientes del despacho como consecuencia de la filosofía y los valores que el despacho oferta.

\section{BIBLIOGRAFÍA}

ALMERA, A. (2010): Introducción al marketing jurídico y el management en los despachos profesionales. Asociación de Marketing Jurídico. Disponible en Internet [URL] www.markjur.com.

Cavero de la PeÑa, I. (2010):» Descubriendo el lado de los negocios en la práctica del derecho». Revista Iuris. Febrero 2010 pp. 27-33.

Cavero de la PeÑa, I. (2011): 11 reglas de marketing jurídico para generar mejores resultados en el despacho. Disponible en Internet [URL] http://www.legaltoday.com/gestion-del-despacho/marke- 
ting/ articulos/11-reglas-de-marketing-juridico-para-generar-mejores-resultados-en-el-despacho.

Domínguez, F. (2002):«El Marketing en la abogacía».Consejo General de la Abogacía Española n ${ }^{\circ} 21$, nueva época, enero-febrero.

Domínguez, F.(2003): El concepto de marketing jurídico. Revista jurídica de lex juris. Disponible en Internet [URL].

http://www.lexjuris.com/revista/opcion1/2003/El\%20concepto\%20de\% 20marketing\%20juridico.htm.

Domínguez, F. (2003):» La planificación estratégica de los bufetes». Abogacía Española n ${ }^{\circ} 25$ abril-junio.

EXPANSión. (2009): la facturación del socio sube un 2,5\%, 7,4 puntos menos que el año pasado (07-07-09).

EXPANSIÓN. (2010): la productividad por abogado se mantiene en la crisis. (11-07-2010).

EXPANSIÓn. (2011): Directivos con todas las de la ley (04-03-2011).

EXPANSIÓN. (2011): Los abogados ven el final del túnel (11-03-2011).

García, J. A.; Pérez de la Manga, M. A. (2009):» ¿se van tus abogados? ¿Sabes por qué?». Revista Economist\&Jurist diciembre-enero $\mathrm{n}^{\mathrm{o}} 126$, pp. 104-108.

GrönRoos, C. (1990): Marketing de Gestión y Servicios. Díaz de Santos.

Hernández GiL, A. (2009): «La abogacía del futuro». Tribuna Abierta Actualidad jurídica-Uría Méndez, no 23 pp. 7-19.

Huete, L. M. (2005): Servicios \& Beneficios. Deusto.

Kotler, P. (1992): Dirección de Marketing, Análisis, planificación, gestión y control. Edit prentice-Hall.

Kotler, P.; Amstrong, G. (2007): Marketing version para latinoamérica. Prentice-Hall.

Kotler, P.; Blomer, H. (2002): Marketing professional services. Prentice-Hall.

La Abogacía Española en Cifras. (2008): edit, Consejo general de la Abogacía Española.

Lambin, J. J. (2003): Márketing estretégico. ESIC. 
Lovelock, C. (1999): Services Marketing a European Perspective. Prentice Hall Europe.

Marcos, F. (2001): «La eficacia de los bufetes de abogados». Economist\&jurist. $\mathrm{n}^{\circ} 47$ (enero-febrero) pp. 82-84.

Martínez Selva, J. M. (1999): Manual de Comunicación Persuasiva para Juristas. La Ley.

Martínez Selva, J. M. (2002): Marketing de Servicios profesionales para la pequeña y mediana empresa. Prentice Hall.

NAVARRo, L. K. (2007): «el marketing en los despachos profesionales; su uso y aplicación». Revista intenauta de práctica jurídica n. ${ }^{\circ} 20$.

Método 3. (2009) Crisis de los despachos de los abogados y marketing jurídico. Professional-letter. Disponible en Internet [URL] www.professional-letter.com.

WinBERG, C. (2009): «los ladrones de tiempo del abogado». Revista Iuris. Marzo pp 25-27. 
